\title{
Chiral vortical effect: Black-hole versus flat-space derivation
}

\author{
G. Yu. Prokhorov $๑^{*}$ and O. V. Teryaev $\circledast^{\dagger}$ \\ Joint Institute for Nuclear Research, Joliot-Curie Street 6, Dubna 141980, Russia \\ and Institute of Theoretical and Experimental Physics, NRC Kurchatov Institute, \\ Bolshaya Cheremushkinskaya 25, Moscow 117218, Russia \\ V. I. Zakharov \\ Institute of Theoretical and Experimental Physics, NRC Kurchatov Institute, \\ Bolshaya Cheremushkinskaya 25, Moscow 117218, Russia \\ and Pacific Quantum Center, Far Eastern Federal University, 10 Ajax Bay, \\ Russky Island, Vladivostok 690950, Russia
}

(Received 9 August 2020; accepted 18 October 2020; published 4 December 2020)

\begin{abstract}
The chiral vortical effect (CVE) was derived first for massless fermions, within the framework of thermal quantum field theory. Recently, a dual description of the CVE, as related to the radiation from the horizon of a rotating black hole was suggested. Generalizing the latter approach to the case of photons, we encounter a crucial factor-of-2 difference from the predictions based on the thermal field theory. To elucidate the reason for this discrepancy, we turn to the limit of large spin $S$ of the massless particles. Within the gravitational approach, the CVE grows as $S^{3}$, while the flat-space relations result in a dependence which is linear in $S$. We also discuss briefly an alternative formulation of the presumed duality between the statistical and gravitational approaches.
\end{abstract}

DOI: 10.1103/PhysRevD.102.121702

\section{GRAVITATIONAL CHIRAL ANOMALY AND CVE FOR SPIN-1/2 PARTICLES}

The chiral vortical effect (CVE) is the flow of chirality of massless particles in a rotating medium along the vector of the angular velocity $\vec{\Omega}$. For a single right-handed Weyl fermion, one finds [1]

$$
\vec{j}_{\mathrm{CVE}}^{N}=\left(\frac{\mu^{2}}{4 \pi^{2}}+\frac{T^{2}}{12}\right) \vec{\Omega},
$$

where $\vec{j}^{N}$ is the particle-number current, $\mu$ is the chemical potential, and $T$ is the temperature.

Remarkably enough, the coefficient in front of the $\mu^{2} \vec{\Omega}$ term turns out to be directly related to the coefficient on the rhs of the chiral anomaly [2],

$$
\partial^{\alpha} j_{\alpha}^{N}=-\frac{1}{32 \pi^{2}} \epsilon^{\alpha \beta \gamma \delta} F_{\alpha \beta} F_{\gamma \delta}
$$

where $F_{\alpha \beta}$ is the electromagnetic field strength tensor.

\footnotetext{
*prokhorov@theor.jinr.ru

teryaev@jinr.ru

‡vzakharov@itep.ru
}

Published by the American Physical Society under the terms of the Creative Commons Attribution 4.0 International license. Further distribution of this work must maintain attribution to the author(s) and the published article's title, journal citation, and DOI. Funded by SCOAP ${ }^{3}$.
The relation, if any, of the $T^{2}$ term in Eq. (1.1) to anomalies remained a kind of mystery until the appearance of Ref. [3]. ${ }^{1}$ The main idea here goes back to Refs. [5-10], which relate the Hawking radiation to the anomalies of quantum field theory. In more detail, it is suggested [3] to consider space-time with a boundary imposed by the horizon of a rotating black hole. Then one can check that near the horizon, the rhs of the gravitational chiral anomaly,

$$
\nabla^{\alpha} j_{\alpha}^{N}=\frac{1}{768 \pi^{2} \sqrt{-g}} \epsilon^{\alpha \beta \gamma \delta} R_{\alpha \beta \rho \sigma} R_{\gamma \delta}^{\rho \sigma}
$$

where $R_{\alpha \beta \gamma \delta}$ is the Riemann tensor, is not vanishing. Far off from the horizon, where the rhs of Eq. (1.3) vanishes, there is a flow of chirality which can be found by integrating the rhs of Eq. (1.3) [3].

This asymptotic current coincides with the $T^{2} \vec{\Omega}$ term in Eq. (4.4), provided that the generic temperature $T$ is replaced by the Hawking temperature $T_{H}$ of the black hole, $T \rightarrow T_{H} \equiv \frac{a_{H}}{2 \pi}$.

Thus, in the case of massless spin- $1 / 2$ particles, there are two complementary ways of deriving the CVE-that is, within the statistical approach in flat space and in terms of black-hole physics. We are considering generalization to the photonic case and obtain a quantitative prediction for

\footnotetext{
${ }^{1}$ For an earlier attempt to relate the CVE to the gravitational anomaly, see Ref. [4].
} 
the CVE of particles with arbitrary spin, based on the gravitational anomaly.

\section{GRAVITATIONAL CHIRAL ANOMALY AND CVE FOR PHOTONS}

As is well known, the chirality of photons is measured by the "charge" $Q_{\text {photon }}^{A}=\int d^{3} x K_{0}$, where the current $K^{\mu}$ is given by

$$
K^{\mu}=\frac{1}{\sqrt{-g}} \epsilon^{\mu \nu \rho \sigma} A_{\nu} \partial_{\rho} A_{\sigma},
$$

where $A_{\mu}$ is the electromagnetic potential. Note that the charge $Q_{\text {photon }}^{A}$ is gauge invariant, unlike the current itself.

The current [Eq. (2.1)] is apparently not conserved, since

$$
\nabla_{\mu} K^{\mu} \equiv \frac{1}{4 \sqrt{-g}} \epsilon^{\mu \nu \rho \sigma} F_{\mu \nu} F_{\rho \sigma}
$$

However, one can demonstrate [11] that naively, the expectation value of the rhs of Eq. (2.2) for photons propagating in external gravitational field vanishes. Moreover, there exists [11-14] the bosonic chiral gravitational anomaly

$$
\left\langle\nabla_{\mu} K^{\mu}\right\rangle=\frac{1}{192 \pi^{2} \sqrt{-g}} \epsilon^{\alpha \beta \gamma \delta} R_{\alpha \beta \rho \sigma} R_{\gamma \delta}^{\rho \sigma} .
$$

Furthermore, Eq. (2.3) suffices to evaluate the chiral vortical effect for photons in terms of the black-hole physics following the logic of Ref. [3].

Indeed, the chiral gravitational anomalies for spin- $1 / 2$ and spin-1 massless particles are proportional to the same $R \tilde{R}$, and the effect of the rotating black hole reduces to a universal geometric factor. We are interested now in the spin dependence of the chiral vortical effect. To elucidate the spin dependence of the CVE, it is convenient to compare fermionic and bosonic fields with an equal number of chiral degrees of freedom - that is, we normalize the photonic case to the case of a Weyl spinor. By comparing Eqs. (1.3) and (2.3), we conclude

$$
\left.\frac{(\mathrm{CVE})_{\text {photons }}}{(\mathrm{CVE})_{\text {Weyl fermions }}}\right|_{\text {black hole }}=4
$$

The problem is that the flat-space derivation suggests rather that the ratio (2.4) is equal to 2 , not 4 .

\section{EVALUATION OF PHOTONIC CVE IN FLAT SPACE}

\section{A. Kubo-type relation}

The most common way to evaluate the CVE in flat space is to use a technique [4] similar to Kubo relations. In more detail, we define the conductivity $\sigma_{\Omega}$ as

$$
\vec{j}_{\mathrm{CVE}}^{N}=\sigma_{\Omega} \vec{\Omega}
$$

Then, $\sigma_{\Omega}$ is given by the retarded two-point Green's function between the current $j_{i}^{N}$ and momentum density $T_{0 j}$ at zero frequency $\omega$ and small momenta $k_{i}$ :

$$
\left.\lim _{k \rightarrow 0} G_{R}(\omega, k)\right|_{\omega=0}=i \epsilon_{i j n} k_{n} \sigma_{\Omega} .
$$

Detailed calculations along these lines in case of charged spin-1/2 particles within thermal field theory can be found, in particular, in Refs. $[15,16]$.

Equation (3.2) can be generalized to the case of photons [15]. The result of calculations can be summarized as

$$
\left.\frac{(\mathrm{CVE})_{\text {photons }}}{(\mathrm{CVE})_{\text {Weyl spinor }}}\right|_{\text {Kubo relation }}=2
$$

Note that Eq. (3.3) differs from Eq. (2.4) by a factor of 2.

Gauge invariance of the results obtained remains a subtle point, since the current $K_{\mu}$ is not explicitly gauge invariant. Gauge invariance could be imposed explicitly by introducing nonlocality. In particular, for photons on a mass shell, the gauge-invariant current reads as

$$
\kappa_{\mu}=(\text { const }) \frac{q_{\mu}}{q^{2}} F_{\alpha \beta} \tilde{F}^{\alpha \beta},
$$

where $q_{\mu}$ is the four-momentum brought in by the current. Note, however, that in the two cases most interesting for applications, the current $\kappa_{\mu}$ reduces in fact to $K_{\mu}$. Namely, evaluation of the charge $\int d^{3} x K_{0}(x)$ assumes taking the limit $q_{i}=0, \omega \rightarrow 0$ in the language of the Fourier transform. In this limit,

$$
\lim _{q_{i}=0, \omega \rightarrow 0} \kappa_{0}=K_{0},
$$

and the charge density $K_{0}$, understood as the component of the Fourier transform with $q_{i} \equiv 0, q_{0} \rightarrow 0$, turns gauge invariant.

Similarly, in the case of the definition (3.2), one considers the limit $\omega=0, q_{3} \rightarrow 0$. In this limit, the nonlocal current $\kappa_{\mu}$ reduces to the component $K_{3}$ :

$$
\lim _{\omega=0, q_{3} \rightarrow 0} \kappa_{3}=K_{3} .
$$

In other words, the component of the Fourier transform of the current defined by Eq. (3.2) is gauge invariant, and the conductivity $\sigma_{\Omega}$ is a physical observable.

\section{B. CVE from the Sommerfeld expansion}

There is another approach to evaluating the CVE, by statistically averaging the matrix element of the corresponding current. One first finds the energy levels as a 
function of the momentum of the particles and of the angular velocity of the medium, and then evaluates the matrix element of the current for each mode.

The latter technique was tried first [1], with the following result, for a Weyl fermion:

$$
\begin{aligned}
J_{\mathrm{CVE}}^{N} & =\frac{1}{4 \pi^{2}} \int_{-\infty}^{\infty} \epsilon^{2} d \epsilon\left(\frac{1}{1+e^{\beta(\epsilon-(\mu+\Omega / 2)}}-\frac{1}{1+e^{\beta(\epsilon-(\mu-\Omega / 2)}}\right) \\
& =\frac{\mu^{2} \Omega}{4 \pi^{2}}+\frac{\Omega^{3}}{48 \pi^{2}}+\frac{T^{2} \Omega}{12}
\end{aligned}
$$

Both the statistical averaging and the use of the Kubo relation result in the same prediction for the CVE in the case of spin- $1 / 2$ particles.

Note that, taken at face value, Eq. (3.7) implies the existence of negative modes at $\epsilon \lesssim \Omega$. However, Eq. (3.7) itself is derived under the assumption that one can expand in $\Omega$, and this assumption is apparently not true at $p \lesssim \Omega$. In other words, the region of small energies $\epsilon$ is to be considered more carefully.

The lowest levels in the rotating system can be found by using the well-known analogy between the magnetic field $\vec{H}$ and the "field of rotation" $\vec{\Omega}$. For massless charged fermions of spin $1 / 2$, the Landau levels are given by

$$
E_{n}= \pm \sqrt{2 H(n+1 / 2)+P_{3}^{2}+H \sigma_{3}}
$$

where $P_{3}$ is the momentum along the magnetic field and $\sigma_{3}= \pm 1$ is the sign of the spin projection onto the direction of the magnetic field. The lowest level, $n=0, P_{3}=0$, $\sigma_{3}=-1$, is the famous zero model responsible for the chiral magnetic effect. In the case of the rotation, however, there is no zero mode for spin $1 / 2$ :

$$
E_{n}>0, \quad \text { spin } 1 / 2, \quad \text { gravity, }
$$

since in the gravitational case, the gyromagnetic ratio is 2 times smaller than in the case of the electromagnetic interaction.

For spin-1 particles in a rotating medium, we would expect that the zero mode comes back:

$$
E_{\min }=0, \quad \text { spin } 1, \quad \text { gravity }
$$

Indeed, the spin is doubled and compensates for the justmentioned loss of the factor of 2. This zero energy is present in the spectrum of photons, as is found by explicit calculation in Ref. [17]. Moreover, introducing a finite radius for the rotating cylinder shifts the lowest-level energy to a small positive value and stabilizes the system.

For higher spins, $S \geq 3 / 2$, the lowest energy level is to be negative:

$$
E_{\min }<0, \quad S \geq 3 / 2, \quad \text { gravity }
$$

and the perturbative vacuum is apparently unstable.

A careful analysis reveals further sources of infrared sensitivity. In particular, it turns out that the chiral vortical current is model independent only as far as it is evaluated on the axis of the rotation, or at the radial coordinate $\rho=0$. On the other hand, as we discussed above [see Eq. (3.2)], evaluation of the CVE assumes that the momentum tends to zero, $q_{i} \rightarrow 0$. And it is only in this limit that we have gauge invariance granted. The conditions $\rho=0$ and $q_{i} \rightarrow 0$ are at least formally in conflict with each other.

To summarize, there are various sources of sensitivity of the global picture to details of the dynamics in the infrared. However, the high-temperature contribution to the CVE might well be protected against these infrared-sensitive effects. Indeed, there is no reason to expect that the two regions, infrared-sensitive and high-temperature ones, give parametrically similar results. Under this assumption, we proceed to consider the higher-spin case.

\section{Limit of large spin of massless particles}

The coefficient in front of the gravitational anomaly grows with spin $S$ of the massless chiral particles as $S^{3}$ for large $S$ [18-20]:

$$
\nabla_{\mu} K_{S}^{\mu}=\frac{(-1)^{2 S}\left(2 S^{3}-S\right)}{192 \pi^{2} \sqrt{-g}} \epsilon^{\mu \nu \rho \sigma} R_{\mu \nu \kappa \lambda} R_{\rho \sigma}{ }^{\kappa \lambda},
$$

where $K_{S}^{\mu}$ is the chiral current for massless particles of spin $S$, an analog of the current $K^{\mu}$ in the photonic case. The current $K_{S}^{\mu}$ can explicitly be constructed in terms of the Pauli-Lubanski pseudovector [20]. Based on the general formula in Eq. (3.12) and following Ref. [3], we predict the $T^{2}$ term in the vortical current:

$\vec{K}_{S}=\frac{(-1)^{2 S}\left(2 S^{3}-S\right)}{3} T^{2} \vec{\Omega} \quad$ (gravitational anomaly).

A striking feature of the prediction (3.13) is its $S^{3}$ dependence. This dependence is not reproduced by thermal field theory in flat space, where the effective coupling $\vec{S} \cdot \vec{\Omega}$ provides that the term linear in $\Omega$ also grows linearly in spin $S$. In particular, according to Ref. [21], the flow of chirality $J_{\chi}$ carried by the spin- $S$ massless particles is given by

$$
\vec{J}_{\chi}=\frac{S \cdot \vec{\Omega}}{\pi^{2}} \sum_{ \pm} \int_{0}^{\infty} f_{ \pm}(p) p d p
$$

where the summation is over the chiral states with projection on the momentum equal to $\pm S ; f_{ \pm}(p)$ are the Bose or 
Fermi distributions, whichever is relevant; and $\mu_{ \pm}$is reserved for nonvanishing chemical potentials.

The predictions (3.13) and (3.14) differ qualitatively. The origin of the disagreement is that in flat space, the effect is controlled primarily by the number of degrees of freedom, which for massless particles does not grow with their spin $S$. The strong $S$ dependence in the case of the gravitational anomaly is apparently due to a specific gravitational interaction with spin. As is noticed in Refs. [18,19], the $S^{3}$ dependence of the gravitational anomaly goes back to the coupling of the Riemann tensor to the spin of the particles:

$$
X=\Sigma^{\mu \nu} R_{\mu \nu}^{a b} \Sigma_{a b}
$$

where $\Sigma^{\mu \nu}$ is the spin operator. Physically, this coupling corresponds to the interaction of the gravitational wave with the quadrupole moment of the particle. ${ }^{2}$

To summarize, the consideration of higher spins elevates the mismatch between the two ways of evaluating the CVE to a qualitative effect. In the next section, we describe briefly another formulation of the statistical approach which provides us with a derivation of the $S^{3}$ dependence.

\section{TEMPERATURE-ACCELERATION, $T \leftrightarrow \frac{a}{2 \pi}$, DUALITY?}

\section{A. Derivation of the Unruh temperature within the statistical approach}

Discovery of the Unruh temperature, or temperature seen by an accelerated observer,

$$
T_{U}=\frac{a}{2 \pi},
$$

established for the first time a kind of equivalence or duality between acceleration $a$ and the temperature $T$. The next step was made in Refs. [5-10]. Here, the basic idea is that at the horizon of the black hole there is an intrinsic right-left asymmetry, since the particles are emitted outwards of the horizon and absorbed inwards. The precise measure of the particle production is provided by the quantum anomalies in terms of the gravitational field, or acceleration on the horizon, $a_{H}$. By matching the flow of the particles far off from the horizon to the radiation from a black body, one is rederiving the Hawking temperature $T_{H}$ :

$$
T_{H}=\frac{a_{H}}{2 \pi} .
$$

Finally, Ref. [3] suggested a similar construction to relate the chiral vortical effect in flat space to the radiation from a rotating black brane.

\footnotetext{
${ }^{2}$ We are grateful to A. I. Vainshtein for this remark.
}

In all these cases, one and the same phenomenon is described either in terms of the statistical theory as a process in equilibrium, or in terms of quantum field theory as a manifestation of an anomaly in the external gravitational field.

There exists another systematic way to relate physics in the equilibrium in flat space to the physics in an external gravitational field and vice versa; for an introduction and further references, see Ref. [22]. On the statistical side, the crucial point is the introduction of the following density operator $\hat{\rho}$ :

$$
\hat{\rho}=\frac{1}{Z} \exp \left(-\beta_{\mu} \hat{P}^{\mu}+\frac{1}{2} \varpi_{\mu \nu} \hat{J}^{\mu \nu}+\xi \hat{Q}\right),
$$

where $\xi=\mu / T, \hat{P}_{\mu}$ is the four-momentum, $\hat{J}^{\mu \nu}$ are generators of the Lorentz transformations, and $\hat{Q}$ is a conserved charge. Moreover, $\varpi_{\mu \nu}=1 / 2\left(\partial_{\nu} \beta_{\mu}-\partial_{\mu} \beta_{\nu}\right)$ is the tensor of the thermal vorticity, and $\beta_{\mu}=u_{\mu} / T$. The operator $\hat{J}^{\mu \nu}$ in Eq. (4.3) can be rewritten as

$$
\hat{J}^{\mu \nu}=u^{\mu} \hat{K}^{\nu}-u^{\nu} \hat{K}^{\mu}-\epsilon^{\mu \nu \rho \sigma} u_{\rho} \hat{J}_{\sigma},
$$

where $\hat{K}^{\mu}$ is the boost operator and $\hat{J}_{\nu}$ is the operator of angular momentum. Furthermore, it is useful to introduce the four-vectors $\alpha_{\mu}, w_{\mu}$ :

$$
\alpha_{\mu}=\varpi_{\mu \nu} u^{\nu}, \quad w_{\mu}=(1 / 2) \epsilon_{\mu \nu \alpha \beta} u^{\nu} \varpi^{\alpha \beta} .
$$

In the rest frame, $T \cdot \alpha^{i}$ and $T \cdot w^{i}$ coincide with the standard three-vectors of acceleration $\vec{a}$ and of the angular velocity $\vec{\Omega}$, respectively.

Note that the boost operators $\hat{K}^{i}$, although conserved, do not commute with the Hamiltonian $\hat{H}$ :

$$
i \frac{d \hat{K}^{i}}{d t}=0, \quad\left[\hat{K}^{i}, \hat{H}\right]=-i \hat{P}^{i},
$$

and the equations in Eq. (4.6) are consistent with each other because of an explicit dependence of $\hat{K}^{i}$ on time [22].

Applications of this technique have proven successful. In particular, the Unruh temperature can be defined now [22] in terms of the energy density $T_{00}$ as a function of acceleration $a$ and of the temperature, $T_{00}(a, T)$ :

$$
\left.T_{00}(a, T)\right|_{T=T_{U}}=0 .
$$

It was demonstrated in Ref. [23] that in case of massless spin- $1 / 2$ and spin- 0 particles, the condition (4.7) allows us to determine the Unruh temperature without introducing any parameter or subtractions.

Note that within the approach of Eq. (4.3), acceleration and temperature are treated as independent variables. To match this freedom on the gravitational side, one considers 
[23] the Rindler space with a conical singularity and defines the acceleration and temperature geometrically. The duality works perfectly well [23]. One can say that the case $\Omega=$ $0,(a, T) \neq 0$ is fully understood in terms of the duality between gravitational and statistical approaches. Here we are considering both $a$ and $\Omega$ to be nonvanishing, and it is a generic feature that the formalism is becoming much more complicated; see in particular Ref. [24].

\section{B. Coupling of acceleration and vorticity to the spin}

The density operator [Eq. (4.3)] which we are exploiting introduces the following effective interaction, specific for the physics of equilibrium:

$$
\delta \hat{H}=-\vec{\Omega} \cdot \overrightarrow{\hat{J}}-\vec{a} \cdot \overrightarrow{\hat{K}}
$$

In the nonrelativistic limit, the part with boost leads to the energy of a particle in a constant gravitational field $a$ [22]:

$$
\hat{H}-a \hat{K}_{z}=m c^{2}+\hat{p}^{2} / 2 m-m a \hat{z} .
$$

More generally, the terms with boost and angular momentum provide that the evolution operator generates a transition between instantaneous inertial rest frames at different time moments, since these transformations in the presence of acceleration are not reduced only to time translations, and they also include Lorentz transformations [25].

In the case of the vectors $\vec{\Omega}$ and $\vec{a}$ parallel to each other, it is straightforward to evaluate the correction to the energy of a chiral state of a spin- $1 / 2$ particle:

$$
\delta E=-\left(\Omega_{z}-i a_{z}\right) \sigma_{z} / 2 .
$$

The reason for "imaginary energy," $\delta E \sim i a / 2$, is the lack of a unitary representation for the boost operator in the case of a chiral multiplet. As a result, one uses an anti-unitary realization of the boost operator, $K^{i} \sim i \sigma^{i}$, which, however, respects commutation relations between the operators. Thermodynamically, this imaginary energy does not make any trouble, since the odd powers of $a$ are canceled out.

Now, the generalization to the case of massless particles of higher spin $S$ is trivial:

$$
\sigma_{z} / 2 \rightarrow S_{z}, \quad \delta E \rightarrow(\vec{\Omega}-i \vec{a}) \vec{S} .
$$

So, each power of $a$ or $\Omega$ is accompanied by a factor of $S$, while the temperature is "unaware" of the spin, since there are 2 degrees of freedom for any massless particle with $S \neq 0$.

On the dimensional grounds for the chiral vortical effect, one has

$$
J_{\mathrm{CVE}} \sim c_{1} T^{2} \Omega+c_{2} a^{2} \Omega,
$$

where the coefficients $c_{1}$ and $c_{2}$ are to be calculated. Following the logic outlined above, we come to the estimates

$$
c_{1} \sim S, \quad c_{2} \sim S^{3} .
$$

The $S^{3}$ dependence of $c_{2}$ matches the prediction of Eq. (3.13) based on the gravitational anomaly, if we use temperature-acceleration duality $T \leftrightarrow \frac{a}{2 \pi}$.

To summarize, straightforward estimates within the statistical approach based on the density operator [Eq. (4.3)] immediately result in a $S^{3}$ dependence of the chiral vortical effect for higher-spin particles.

\section{CONCLUSIONS}

Two approaches have been tried to evaluate the chiral vortical effect (CVE) for massless spin-1/2 particles, with identical results obtained. The first approach is based on statistical averaging of the matrix elements of the axial current. The other one relates the CVE to the chiral gravitational anomaly. The latter approach can immediately be generalized to the case of the arbitrary spin of particles interacting with an external gravitational field.

The prediction for the photonic CVE obtained in this way can be compared with the results obtained within the statistical approach. In particular, application of the Kubotype relation to evaluate the photonic CVE gives an answer $[15,16]$ which differs by a factor of 2 from the gravitational approach. However, there is no consensus yet in the literature concerning the value of the vortical conductivity $\sigma_{\Omega}^{\gamma}$. The source of uncertainty is apparently dependence on the regularization procedure in the infrared. Thus, it is not ruled out that the final result for photons would agree with the prediction of the gravitational approach.

To have a clearer case, we turn to consideration of the limit of large spin $S$ of the constituents. In this case, the predictions for the vortical conductivity differ qualitatively: gravitational anomaly predicts $S^{3}$ dependence, while thermal field theory leads to linear dependence on $S$.

We also try an alternative statistical approach based on the use of the density operator [Eq. (4.3)]. This version of the statistical approach does reproduce the $S^{3}$ dependence of the vortical effect in an accelerated medium.

There are many questions left open. The main reservation concerning the status of the results obtained is that theories involving massless particle with large spin $S$ have intrinsic problems and difficulties.

\section{ACKNOWLEDGMENTS}

The authors are thankful to P. G. Mitkin, A. V. Sadofyev, A. I. Vainshtein, and A. I. Vasiliev for valuable discussions and to J. Erdmenger and R. Banerjee for communications. The work is partially supported by RFBR Grants No. 1802-40056 and No. 0657-2020-0015 of the Ministry of Science and Higher Education of Russia. 
[1] A. Vilenkin, Quantum field theory at finite temperature in a rotating system, Phys. Rev. D 21, 2260 (1980).

[2] D. T. Son and P. Surowka, Hydrodynamics with Triangle Anomalies, Phys. Rev. Lett. 103, 191601 (2009).

[3] M. Stone and J. Kim, Mixed anomalies: Chiral vortical effect and the Sommerfeld expansion, Phys. Rev. D 98, 025012 (2018).

[4] K. Landsteiner, Eu. Megias, and F. Pena-Benitez, Gravitational Anomaly and Transport, Phys. Rev. Lett. 107, 021601 (2011).

[5] S. P. Robinson and F. Wilczek, Relationship between Hawking Radiation and Gravitational Anomalies, Phys. Rev. Lett. 95, 011303 (2005).

[6] S. Iso, H. Umetsu, and F. Wilczek, Hawking Radiation from Charged Black Holes via Gauge and Gravitational Anomalies, Phys. Rev. Lett. 96, 151302 (2006).

[7] S. Iso, H. Umetsu, and F. Wilczek, Anomalies, Hawking radiations and regularity in rotating black holes, Phys. Rev. D 74, 044017 (2006).

[8] R. Banerjee and S. Kulkarni, Hawking radiation and covariant anomalies, Phys. Rev. D 77, 024018 (2008).

[9] R. Banerjee and S. Kulkarni, Hawking radiation, effective actions and covariant boundary conditions, Phys. Lett. B 659, 827 (2008).

[10] R. Banerjee and S. Kulkarni, Hawking radiation, covariant boundary conditions and vacuum states, Phys. Rev. D 79, 084035 (2009).

[11] A. I. Vainshtein, A. D. Dolgov, V. I. Zakharov, and I. B. Khriplovich, Chiral photon current and its anomaly in a gravitational field, Zh. Eksp. Teor. Fiz. 94, 54 (1988) [Sov. Phys. JETP 67, 1326 (1988)].

[12] I. Agullo, A. del Rio, and J. Navarro-Salas, Electromagnetic Duality Anomaly in Curved Spacetimes, Phys. Rev. Lett. 118, 111301 (2017).

[13] A. Avkhadiev and A. V. Sadofyev, Chiral vortical effect for bosons, Phys. Rev. D 96, 045015 (2017).
[14] J. Erdmenger, Gravitational axial anomaly for fourdimensional conformal field theories, Nucl. Phys. B562, 315 (1999).

[15] S. Golkar and D. T. Son, (Non)-renormalization of the chiral vortical effect coefficient, J. High Energy Phys. 02 (2015) 169.

[16] D.-F. Hou, H. Liu, and H.-C. Ren, A possible higher order correction to the vortical conductivity in a gauge field plasma, Phys. Rev. D 86, 121703 (2012).

[17] M. N. Chernodub, A. Cortijo, and K. Landsteiner, Zilch vortical effect, Phys. Rev. D 98, 065016 (2018).

[18] S. M. Christensen and M. J. Duff, New gravitational index theorems and supertheorems, Nucl. Phys. B154, 301 (1979).

[19] M. J. Duff, Supergravity 81, Proceedings of 1st School on Supergravity, edited by S. Ferrara and J. G. Taylor (Cambridge University Press, Cambridge, England, 1982), preprint Ref. TH.3232-CERN.

[20] A. D. Dolgov, I. B. Khriplovich, A. I. Vainshtein, and V. I. Zakharov, Photonic chiral current and its anomaly in a gravitational field, Nucl. Phys. B315, 138 (1989).

[21] X. G. Huang and A. V. Sadofyev, Chiral vortical effect for an arbitrary spin, J. High Energy Phys. 03 (2019) 084.

[22] F. Becattini, Thermodynamic equilibrium with acceleration and the Unruh effect, Phys. Rev. D 97, 085013 (2018).

[23] G. Y. Prokhorov, O. V Teryaev, and V. I. Zakharov, Unruh effect universality: Emergent conical geometry from density operator, J. High Energy Phys. 03 (2020) 137.

[24] J. D. Brown, E. A. Martinez, and J. W. York, Jr., Complex Kerr-Newman Geometry and Black Hole Thermodynamics, Phys. Rev. Lett. 66, 2281 (1991).

[25] J. I. Korsbakken and J. M. Leinaas, The Fulling-Unruh effect in general stationary accelerated frames, Phys. Rev. D 70, 084016 (2004). 